\title{
Comment to the letter of Dr. Yeon-Suk Kim et al.
}

\author{
Rudy Leon De Wilde ${ }^{1}$
}

Received: 13 February 2018 / Accepted: 28 February 2018 / Published online: 12 March 2018

c) Springer-Verlag GmbH Germany, part of Springer Nature 2018

Dear Editor,

The authors of "Pathobiology of myomatosis uteri: the underlying knowledge to support our clinical practice" want to thank Yeon-Suk Kim, Tae-Hee Kim, Hae-Hyeog Lee and Kisung Song for the valuable comments on their manuscript.

The performed whole-genome sequencing (WGS) seems superior in detecting mutations in non-coding regions, possibly leading to improved understanding of the pathogenesis in myoma formation. WGS, conducted in a large myoma, supports the knowledge of the proposed selected genes, harboring single-nucleotide polymorphisms.

IL-37, a suppressor of immune response, could play a crucial role in the multi-factorial mechanism of large myoma development and growth. The documented results require further research concerning the expression of myomaassociated inflammatory interleukins and will eventually explain the failure of clinical therapies targeting monofactorial pathways.
Rudy Leon De Wilde

rudy-leon.dewilde@pius-hospital.de

1 Clinic of Gynecology, Obstetrics and Gynecological

Oncology, Pius Hospital University Hospital

for Gynecology, Carl von Ossietzky University Oldenburg,

Oldenburg, Germany 